\title{
Influence of the Local Field and Dipole-Dipole Interactions on the Spectral Characteristics of Simple Metals and Their Nanoparticles
}

\author{
I. I. Shaganov \\ Vavilov State Optical Institute \\ Kevin Berwick \\ Technological University Dublin, kevin.berwick@tudublin.ie \\ T. S. Perova \\ Trinity College Dublin, Ireland
}

Follow this and additional works at: https://arrow.tudublin.ie/engscheleart2

Part of the Electrical and Computer Engineering Commons

\section{Recommended Citation}

Shaganov, I.I., Berwick, K. \& Perova, T.S. Influence of the Local Field and Dipole-Dipole Interactions on the Spectral Characteristics of Simple Metals and Their Nanoparticles. Plasmonics 14, 1443-1451 (2019).

DOI: 10.1007/s11468-019-00939-4

This Article is brought to you for free and open access by the School of Electrical and Electronic Engineering at ARROW@TU Dublin. It has been accepted for inclusion in Articles by an authorized administrator of ARROW@TU Dublin. For more information, please contact arrow.admin@tudublin.ie, aisling.coyne@tudublin.ie, gerard.connolly@tudublin.ie.

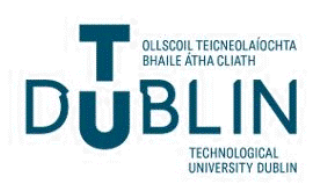




\title{
Influence of the Local Field and Dipole-Dipole Interactions on the Spectral Characteristics of Simple Metals and Their Nanoparticles
}

\author{
I. I. Shaganov ${ }^{1} \cdot$ K. Berwick ${ }^{2} \cdot$ T. S. Perova ${ }^{3}$ (I) \\ Received: 27 September 2018 / Accepted: 11 March 2019 / Published online: 29 March 2019 \\ (C) Springer Science+Business Media, LLC, part of Springer Nature 2019
}

\begin{abstract}
The effect of dipole-dipole interactions of free electrons on the spectral characteristics of simple metals and their nanoparticles is analyzed using Drude theory and the model of the Lorentz local field. It is established that accounting for the dispersion of a local field under conditions of one-dimensional (1D) confinement based on the optical constants of the bulk metal allows the determination of its spectral micro-characteristics in the frequency region of the longitudinal collective motions of the free electrons. This corresponds to the spectra of the dielectric losses of bulk plasma oscillations. A similar procedure for threedimensional (3D) confinement produces the spectrum of dielectric losses at the frequency of localized plasma oscillations. Using a number of simple metal examples, viz., $\mathrm{Li}, \mathrm{Na}$, and $\mathrm{K}$, and also $\mathrm{Al}, \mathrm{Be}$, and $\mathrm{Mg}$, it is shown that the frequencies of volume and localized plasma oscillations obtained from a model of dispersion of the local field in the long-wave limit are in good agreement with the actual frequencies of the plasma oscillations of the corresponding metals and the absorption maxima of their nanoparticles with a radius of 2-20 nm. It is shown that the frequencies of the main mode of longitudinal plasma oscillations and the absorption frequency of localized plasmons are well described using the dynamic theory of crystal lattice vibrations.
\end{abstract}

Keywords Bulk plasma oscillation $\cdot$ Localized plasmon $\cdot$ Metal nanoparticles $\cdot$ Local-field effect

\section{Introduction}

The optical properties of metals and their nanoparticles currently attract much attention due to the rapid development of plasmonics, a new and promising field of applied physics and nanotechnology [1-5]. The possibility of excitation of localized plasmons in nanoparticles of $\mathrm{Au}, \mathrm{Ag}, \mathrm{Cu}, \mathrm{Al}$, and other metals is of great practical interest for obtaining media with unique optical properties [3] and the development of novel plasmonic devices [6-9]. The plasmonic properties of noble metals (NMs) such as $\mathrm{Au}$ and $\mathrm{Ag}$ and to a lesser extent, $\mathrm{Cu}$, which possess a localized plasmonic resonance in the visible range, have been investigated very actively over the last three decades, both theoretically and experimentally. These investigations, together

\section{T. S. Perova}

perovat@tcd.ie

Vavilov State Optical Institute, St.-Petersburg, Russia 199034

2 School of Electrical and Electronic Engineering, Dublin Institute of Technology, Dublin 8, Ireland

3 Department of Electronic and Electrical Engineering, Trinity College Dublin, The University of Dublin, Dublin 2, Ireland with many thousands of original journal papers, have been summarized in numerous books and review articles, for example, [1-12]. This is because the NMs, in particular Au and Ag, have been used technologically in a variety of applications. These include medical diagnostics and therapeutics technologies [13-15], drug delivery [16], manipulation of light for photovoltaics and solar energy technologies [6, 17], extreme UV lasers [18], and modern topic nowadays-magneto-plasmonic nanoantennas [19] — among others.

A deeper understanding of the nature of the formation of the complex dielectric function of the metal, $\varepsilon(\omega)$, remains an important requirement for further progress in creating new metamaterials [3]. At present, the optical properties of metals and data on their dielectric function over a wide spectral region are available for an increasing number of metallic media [20-22]. An understanding of the impact of free and bound electrons on the optical characteristics of metals is a necessary condition for minimizing dielectric losses in waveguides, resonators, solar cells, and other plasmonic devices [1-3].

In this paper, using Drude theory and Lorentz's local field model, as well as the results from the theory of dipole-dipole interactions, an analysis is made of the effect of free electrons on the spectroscopic characteristics 
of simple metals and their nanoparticles in the frequency range of volume and localized plasma oscillations. The results indicate that the interactions of free electrons in simple metals can be described using the theory of dipole-dipole interactions while accounting for the dispersion of the local field in a condensed medium. It is shown that the maxima of the dielectric loss spectra of $\mathrm{Li}, \mathrm{Na}$, and $\mathrm{K}$, as well as $\mathrm{Al}, \mathrm{Be}$, and $\mathrm{Mg}$, in the spectral region of bulk and localized plasmons, calculated using the Lorentz field from the optical constants of the bulk metal, as well as the dynamic theory of the crystal lattice and the influence of dipole-dipole interactions, are in good agreement with known values of the plasma frequencies of these metals and the absorption maxima of the corresponding spherical nanoparticles with dimensions much smaller than the wavelength. The absorption maxima were obtained from Mie theory calculations and from experimental data on the absorption spectra of spherical nanoparticles.

\section{Theoretical Considerations}

\section{Drude-Lorentz Model}

The optical response of a metal to an external electromagnetic field is largely due to the motion of free electrons within the crystal structure of the metal. In the Drude model, describing a metal as a vibration of a free electron gas filling the space around a positively charged ion core, these electrons move freely in the crystal lattice without any restoring force. This corresponds to the fact that the resonance frequency of an individual electron is zero. The electron gas experiences longitudinal plasma oscillations, which are collective displacements of the elementary volume of the electron gas as a whole with respect to the positive ion background. This displacement creates an electric field $E=$ $4 \pi N e x$, acting as a restoring force, which is neutralized by fields induced by the polarization of the medium due to Coulomb dipole-dipole interactions. The equation of motion of a unit volume of an electron gas in this case has the form [23]

$\frac{d^{2} x}{d t^{2}}+\omega_{\mathrm{p}}^{2} x=0$

where $\omega_{\mathrm{p}}=\left(4 \pi N e^{2} / m\right)^{1 / 2}$ is the plasma frequency.

This equation has the same form as the equation of motion of a harmonic oscillator with an eigen, or intrinsic, frequency $\omega_{\mathrm{p}}$. So, the consideration of the interactions of free electrons in a metal with an electromagnetic field $E(\omega)$ using the Lorentz oscillator model is completely justified. The frequency $\omega_{\mathrm{p}}$, known as the plasma frequency, is the frequency of the collective longitudinal vibrations of free electrons. It is the most important parameter of the Drude expression for the complex dielectric permittivity of a metal

$\varepsilon_{\text {intra }}(\omega)=1+\frac{\omega_{\mathrm{p}}^{2}}{\left(\omega^{2}+i \gamma \omega\right)}$,

where $\gamma$ is the damping constant, which takes into account the frequency of collisions of the electrons.

So, further consideration of the interactions of free electrons in a metal with an electromagnetic field $E(\omega)$ using the Lorentz oscillator model is fully justified. It should be noted that the effect of interband transitions as well as the ionic lattice of a metal in the Drude model is not taken into account. The contribution of interband transitions and the influence of the ion core can be considered by introducing an additional term in Eq. (2), which, in a typical Lorentz oscillator, has the form

$\varepsilon_{\text {inter }}(\omega)=\sum \frac{F_{i} \omega_{i}^{2}}{\left(\omega_{i}^{2}-\omega^{2}-i \omega \Gamma_{i}\right)}$

where $\omega_{i}$ is the resonant frequency of the $i^{\text {th }}$ interband transition, and $F_{i}$ and $\Gamma_{i}$ are the strength of the oscillator and the damping coefficient respectively.

A general expression for the spectrum of the dielectric function of the metal can then be represented as the DrudeLorentz relation

$\varepsilon(\omega)=\varepsilon_{\infty}-\frac{\omega_{\mathrm{p}}^{2}}{\left(\omega^{2}+i \gamma \omega\right)}$,

where $\varepsilon_{\infty}$ takes into account the contribution of highfrequency interband transitions, described by Eq. (3). It should be noted that in the Drude-Lorentz free-electron model it is assumed that the local field acting on the electron is equal to the mean field in the metal $E_{\mathrm{loc}}(\omega)=E_{\mathrm{av}}(\omega)$. The influence of the ionic lattice in this case is considered as a homogeneous background of the positive charge, and the heterogeneity of the lattice potential is not taken into account. Thus, considering the band structure of the metal only requires the replacement of the electron mass $m$ by its effective value $m^{*}$.

The periodicity of the ionic structure, however, leads to an inhomogeneity of both the ion potential and the potential of the free electrons. In this regard, even the local effective field, $E_{\text {eff }}(\omega)$, averaged over the cell of the crystal is not equal to the average macroscopic field, $E_{\mathrm{av}}(\omega)$ [24]. The first attempt to use the local field correction in the analysis of the effect of Coulomb dipole-dipole interactions on the spectroscopic characteristics of noble metals and their nanoparticles was undertaken in Ref. [25]. We shall now consider the results of correcting the local field with reference to the spectra of simple metals in the region of the volume plasma oscillation frequencies. 


\section{Accounting for the Dispersion of the Local Field in a Condensed Medium}

In the model of a harmonic oscillator under a local field in a condensed medium $\left(E_{\text {loc }}\right)$, a complete microscopic field is assumed at the point where the oscillator is located, minus the field created by it. The field $E_{\mathrm{loc}}$, the local Lorentz field acting on a separate oscillator due to all other oscillators in the system, is related to the average macroscopic field $(E)$ in the given medium by the relationship.

$E_{\mathrm{loc}}(\omega)=E(\omega) \frac{[\varepsilon(\omega)+2]}{3}$,

where $\varepsilon(\omega)=\varepsilon_{1}(\omega)-i \varepsilon_{2}(\omega)$ is the complex dielectric permittivity of the medium at a given frequency. With metals where the localization of electrons is absent, Eq. (5) characterizes the average local field in the microvolume of the electron gas created by the external field and the fields of the surrounding charges formed by a neighboring electron cloud and an ion lattice. Equation (5) is the basis of the effective local field method used in this paper. This method, proposed by the authors of Ref. [26] more than half a century ago, turned out to be convenient for analyzing the influence of dipole-dipole interactions on the absorption spectra of various condensed media. It is based on a comparison of the absorption spectrum or dielectric loss spectrum of a condensed medium $\varepsilon_{2}(\omega)$ and its microscopic analogue $\varepsilon_{2}^{\text {mic }}(\omega)$ calculated in accordance with the expression

$\varepsilon_{2}^{\mathrm{mic}}(\omega)=\varepsilon_{2}(\omega) \theta(\omega)$,

where $\theta(\omega)$ is the local field correction factor whose spectral behavior as determined by the Lorentz model is described as

$\theta(\omega)=\frac{9}{|\varepsilon(\omega)+2|^{2}}$.

Using the correction above allows one to obtain the spec$\operatorname{trum} \varepsilon_{2}^{\mathrm{mic}}(\omega)$ from which the influence of the optical polarization of the condensed medium or, in other words, the contribution of dipole-dipole interactions in the frequency region of the resonance under consideration is excluded. By comparing the frequencies and intensities of the spectra $\varepsilon_{2}(\omega)$ and $\varepsilon_{2}^{\text {mic }}(\omega)$, we can draw conclusions about the role of dipoledipole interactions in the case under consideration. The utility of the method has been demonstrated during the analysis of the spectral characteristics of various condensed media in Refs. [27-30]. It should be noted that the applicability of the considerations above is not limited to molecular crystalline, liquid, and amorphous media, where the absorbing centers can be regarded as a set of individual oscillators.

Another important example is the calculation of the microcharacteristics of alkali-halide crystals in the frequency range of fundamental lattice vibrations, see, for example, Ref. [31]. In this case, the frequency of the maximum of the spectrum, $\varepsilon_{2}^{\text {mic }}(\omega)$, corresponds to the frequency of the fundamental mode of the oscillations of the microregion of a crystal much larger than the lattice constant, but much smaller than the wavelength of the probing radiation. The frequency in question is near the frequency, $\omega_{\mathrm{F}}$, satisfying the Fröhlich's condition [32], namely $\varepsilon_{1}\left(\omega_{\mathrm{F}}\right)=-2$ and agrees with the intrinsic frequency of the lattice vibrations calculated from the elastic constants of the crystal. The data obtained shows that Eq. (6) characterizes the spectrum of dielectric losses of a spherical particle of a condensed medium with a diameter $d<<\lambda$, with its own spectral characteristics.

The general nature of the concepts considered is confirmed by results from an analysis of the influence of dipole-dipole interactions on the plasmon absorption spectra of noble metal nanoparticles and their colloidal solutions [25, 33]. Thus, in the case of $\mathrm{Au}, \mathrm{Ag}$, and $\mathrm{Cu}$ [25], the frequencies of the calculated spectra $\varepsilon_{2}^{\text {mic }}(\omega)$ are close to the frequency maximum of the absorption spectra of the corresponding nanoparticles with a radius of 2-30 $\mathrm{nm}$. The frequencies are also in good agreement with estimates from the theory of dipole-dipole interactions. It should be noted that Eq. (7) corresponds to the limiting case of 3D dielectric confinement, when the dimensions of the condensed medium decrease to a sphere with a diameter much less than the wavelength. In the general case of 1D, 2D, and $3 \mathrm{D}$ confinement, Eq. (7) can be expressed in the following form [30];

$\theta_{m}(\omega)=\frac{1}{|1+(\varepsilon(\omega)-1) / m|^{2}}$,

where $m=1,2$, and 3, respectively. As will be shown below, one-dimensional confinement corresponds to losses at the frequency of the longitudinal optical vibrations of the condensed medium under consideration.

\section{Longitudinal Vibrations in Condensed Medium}

The longitudinal oscillations of electrons in a metal are analogous to the longitudinal optical vibrations of a lattice of alkali-halide crystals [34]. In this case, the splitting of longitudinal (LO) and transverse (TO) optical modes due to long-range Coulomb dipole-dipole interactions [35] is observed in the IR spectra of these crystals. The effect of optical polarization and the consequent dipole-dipole interactions generates opposite shifts in the frequencies of the longitudinal $\omega_{\mathrm{LO}}$ and transverse $\omega_{\text {TO }}$ optical vibrational modes relative to the intrinsic frequency of the oscillations of the crystal $\omega_{0}$, also known as the bare frequency.

As noted above, this frequency corresponds to the maximum of the spectrum, $\varepsilon_{2}^{\text {mic }}(\omega)$, obtained for 3D confinement. 
For 1D confinement, the frequency of the spectrum, $\varepsilon_{2}^{\text {mic }}(\omega)$, coincides with the frequency of longitudinal vibrations $\left(\omega_{\mathrm{LO}}\right)$ and corresponds to the spectrum of a thin film with thickness $d$ $<\lambda$ and with the external field strength vector $E(\omega)$ perpendicular to its plane. The influence of dipole-dipole interactions on the frequency shifts of longitudinal and transverse oscillations is well described using the theory of collective excitations and the dynamical theory of crystal lattice vibrations. According to Born and Huang [35] and Bonneville [36], the corresponding expressions for the frequencies of the transverse $\nu_{\mathrm{TO}}$ and longitudinal $\nu_{\mathrm{LO}}$ modes have the form

$\nu_{\mathrm{TO}}=\left[\nu_{0}^{2}-\left(\frac{N f e^{2}}{3 \pi c^{2}}\right) \cdot \frac{\varepsilon_{\infty}+2}{3}\right]^{1 / 2}$
$\nu_{\mathrm{LO}}=\left[\nu_{0}^{2}+\left(\frac{2 N f e^{2}}{3 \pi c^{2}}\right) \cdot \frac{\varepsilon_{\infty}+2}{3 \varepsilon_{\infty}}\right]^{1 / 2}$

where $\nu_{0}$ is the frequency of the microscopic oscillator (the intrinsic frequency of the lattice vibrations in the absence of the medium's polarization effect) per centimeter, $f$ is the strength of the microscopic oscillator, $e$ is the charge of the electron, $c$ is the speed of light, and $N$ is the number of ion pairs per cubic centimeter. We will use the wavenumber $\nu$, as used in molecular spectroscopy,
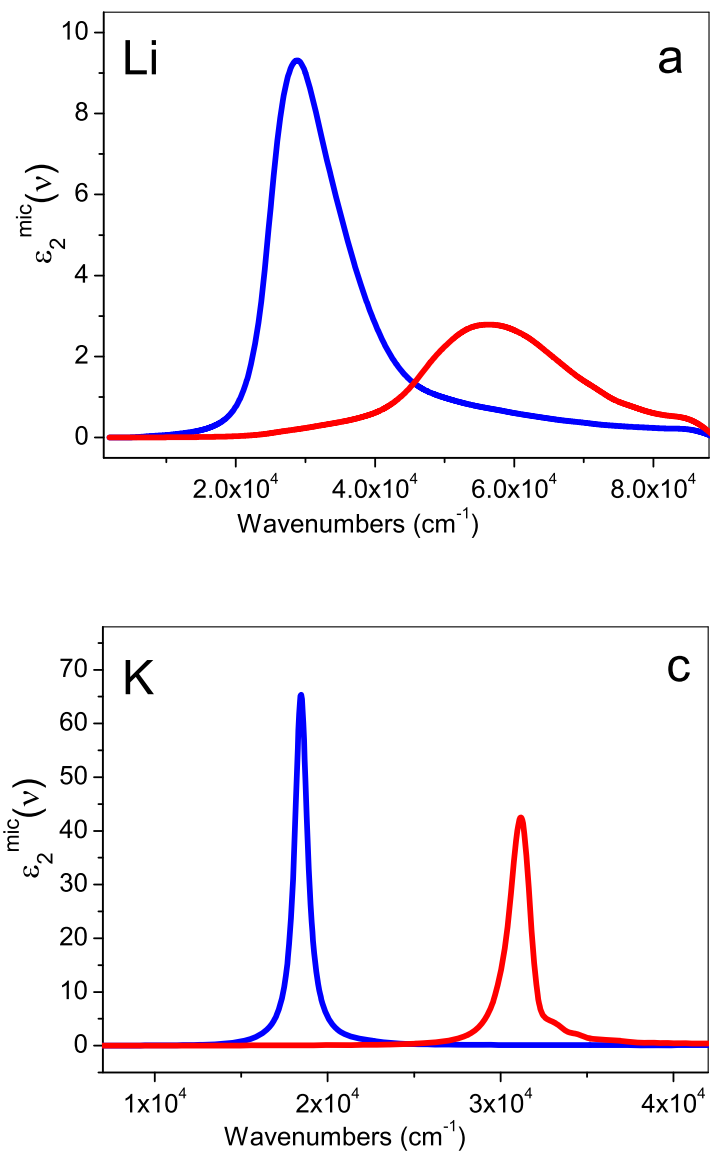

Fig. 1 Calculated spectra $\varepsilon_{2}^{\mathrm{mic}}(\nu)$ for 3D confinement (blue line) and for $1 \mathrm{D}$ confinement (red line) for a Li, b $\mathrm{Na}$, $\mathbf{c} \mathrm{K}$, and $\mathbf{d ~ A l}$

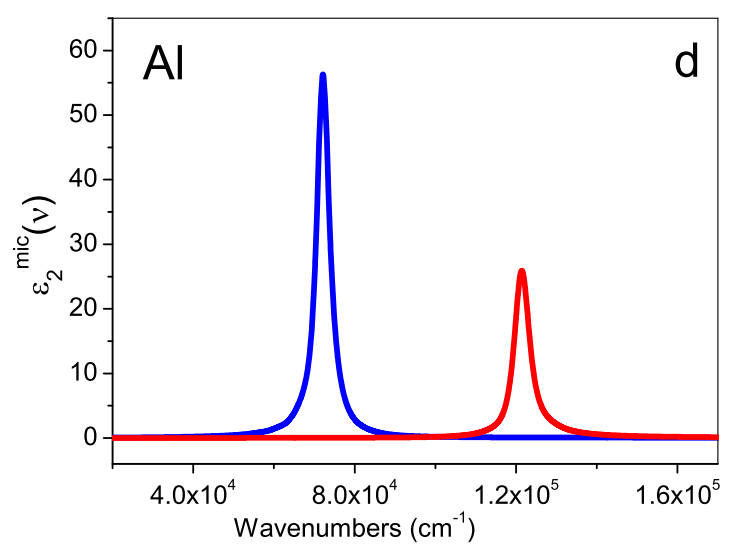

instead of $\omega$ below, but will continue to refer to it as frequency, measured per centimeter and related to $\omega$ as $\nu=\omega / 2 \pi c$. As shown earlier, the force of the microscopic oscillator is determined by the integrated intensity of the corrected spectrum $\varepsilon_{2}^{\text {mic }}(\nu)$ as

$f=\left(\frac{2 m c^{2}}{N e^{2}}\right) \nu_{3 \mathrm{D}}^{\max } \int \varepsilon_{2}^{\mathrm{mic}}(\nu) d \nu$.

Where $\nu_{3 \mathrm{D}}^{\max }$ is the frequency of the maximum of the spectrum $\varepsilon_{2}^{\text {mic }}(\nu)$, calculated under $3 \mathrm{D}$ confinement.

Equations (9) and (10) are a good description of the shift in the transverse and longitudinal modes with respect to the intrinsic vibration frequency of the crystal and, accordingly, the total LO-TO splitting [35, 36]. According to the Drude model for the metal, the resonance frequency of the transverse vibrations of free electrons $\nu_{\mathrm{TO}}$ is zero. Equation (9) is transformed into a form that coincides with the expression for the shift $\left(\Delta \nu_{\mathrm{dyn}}\right)$ of the maximum of the macroscopic spectrum $\varepsilon_{2}^{\text {bulk }}(\omega)$ relative to the intrinsic frequency of the microscopic oscillator $\nu_{3 \mathrm{D}}^{\max }$ due to the effect of the medium polarization from the theory of dipole-dipole interactions. This shift, as shown in [25], actually coincides with the value of the intrinsic frequency, i.e., $\Delta \nu_{\mathrm{dyn}}=\nu_{0}$

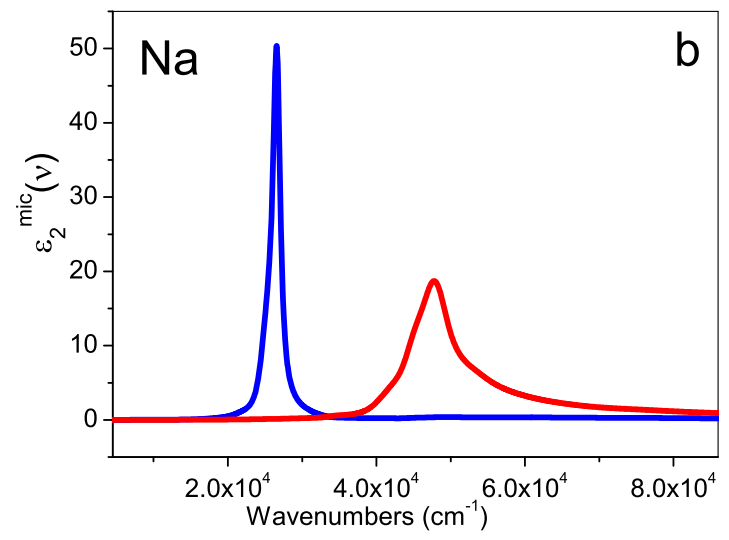


$\nu_{0}=\left[\frac{\left(\frac{N f e^{2}}{3 \pi m c^{2}}\right)\left(\varepsilon_{\infty}+2\right)}{3}\right]^{1 / 2}$

From Eqs. (11) and (12), it follows that $\nu_{0}$ can be expressed as

$\nu_{0}=\left[\frac{2 \nu_{3 D}^{\max }\left(\varepsilon_{\infty}+2\right) I_{3 D}}{9 \pi}\right]^{1 / 2}$

where $I_{3 D}=\int \varepsilon_{2}^{\operatorname{mic}}(\nu) d \nu$.

From Eqs. (9) and (10), it also follows that the frequency of the longitudinal mode can be represented in the form

$\nu_{\mathrm{LO}}=\left[\frac{3 \nu_{0}^{2}\left(\varepsilon_{\infty}+2\right)}{3 \varepsilon_{\infty}}\right]^{1 / 2}$

It is straightforward to see that when the value of the background dielectric permittivity is close to unity $\left(\varepsilon_{\infty} \approx 1\right)$, Eq. (13) coincides with the relationship between the plasma volume frequency $\nu_{\mathrm{P}}$ and the frequency of the localized plasmon for the spherical particle, $\nu_{\mathrm{S}}[5,37]$

$\nu_{\mathrm{P}}=\left(3 \nu_{S}^{2}\right)^{1 / 2}=\nu_{S} \sqrt{3}$.
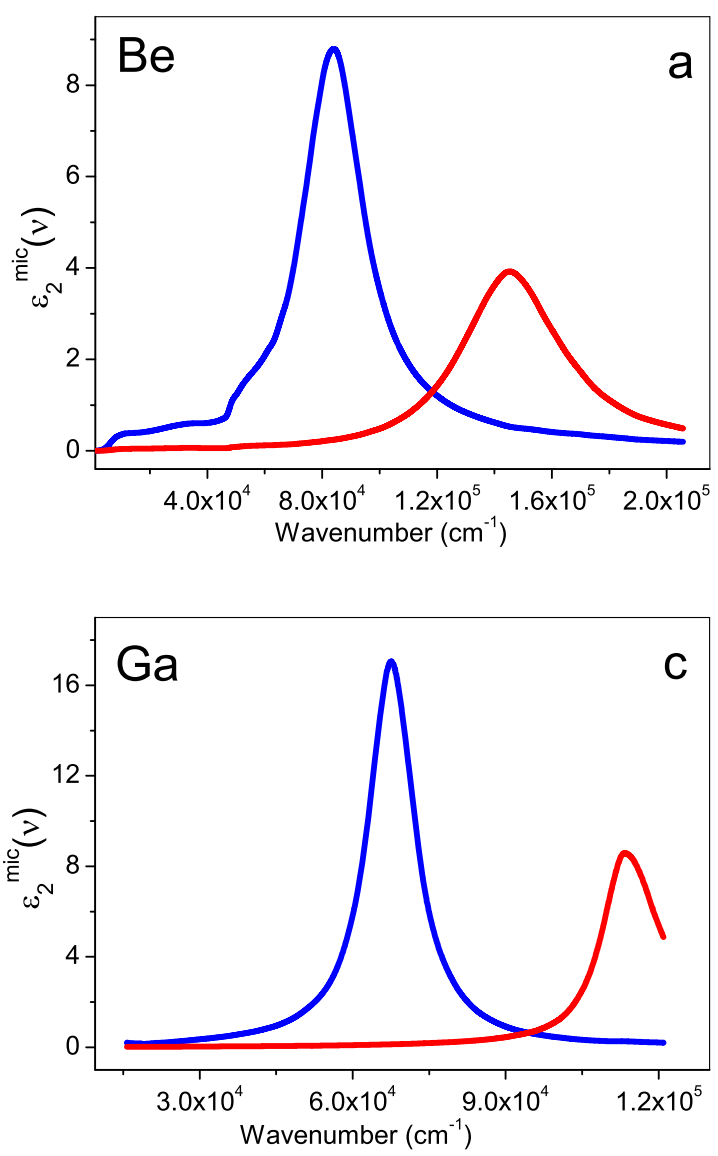

Expressions (12) and (13) were used in the analysis of the dielectric loss spectra $\varepsilon_{2}^{\text {mic }}(\nu)$ of a number of simple metals calculated using the expressions (6) and (8) for 3D (denoted as $\left.\varepsilon_{2}^{\text {mic }}\left(\nu_{3 \mathrm{D}}\right)\right)$ and $1 \mathrm{D}$ (denoted as $\left.\varepsilon_{2}^{\text {mic }}\left(\nu_{1 \mathrm{D}}\right)\right)$ confinement, i.e., for the cases of $m=3$ and $m=1$ in Eq. (8). For these calculations, data for the optical constants $(n(\nu)$ and $k(\nu))$ of bulk metal were taken from different sources, from Ref. [38] for $\mathrm{Al}$, from Ref. [39] for $\mathrm{K}, \mathrm{Li}, \mathrm{Na}$, and $\mathrm{Be}$, from Ref. [40] for $\mathrm{Mg}$ and In, and from Ref. [41] for Ga. The spectra $\varepsilon_{2}^{\text {mic }}\left(\nu_{3 \mathrm{D}}\right)$ and $\varepsilon_{2}^{\text {mic }}\left(\nu_{1 \mathrm{D}}\right)$ calculated for $\mathrm{Li}, \mathrm{Na}$, and $\mathrm{K}$, as well as for $\mathrm{Al}$, $\mathrm{Be}$ and $\mathrm{Mg}$, are shown in Figs. 1 and 2, respectively. The frequencies of the absorption maxima of these spectra are summarized in Table 1.

From Table 1, the frequencies of the maxima of the $\varepsilon_{2}^{\text {mic }}$ $\left(\nu_{3 \mathrm{D}}\right)$ spectra (column VI), corresponding to the absorption of micro-regions of a metal with dimensions $d<\lambda$, are observed near the Fröhlich frequency, $v_{\mathrm{F}}$, at $\operatorname{Re} \varepsilon\left(v_{\mathrm{F}}\right)=-2$, the value of which, estimated from the experimental spectrum $\varepsilon_{2}(\nu)$, is given in column XII. They also correlate well with the absorption frequencies of the corresponding spherical nanoparticles with a radius of $\sim 2-20 \mathrm{~nm}$, calculated in this work, using a simplified Mie formula [5], column X, and using more advanced calculations and some experimental data from Refs. [42-44], presented in column XI. At the same time,
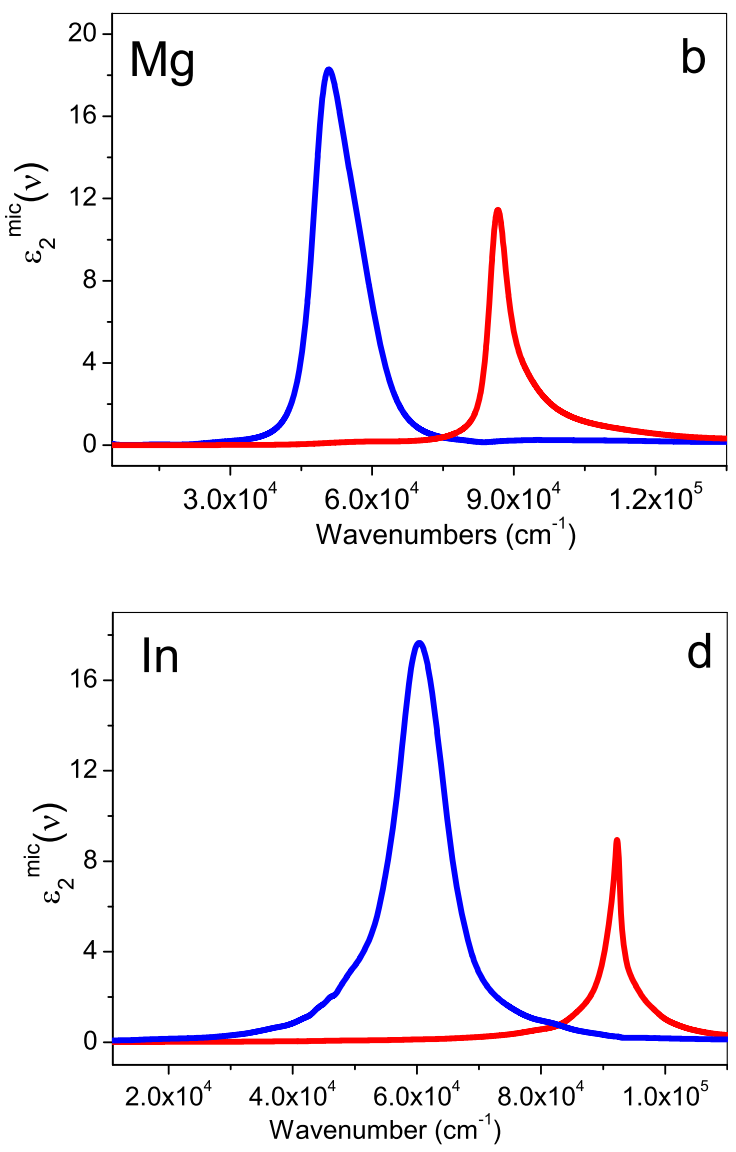

Fig. 2 Calculated spectra $\varepsilon_{2}^{\text {mic }}(\nu)$ for 3D confinement (blue line) and for 1D confinement (red line) for a Be, b Mg, $\mathbf{c} \mathrm{Ga}$, and $\mathbf{d}$ In 


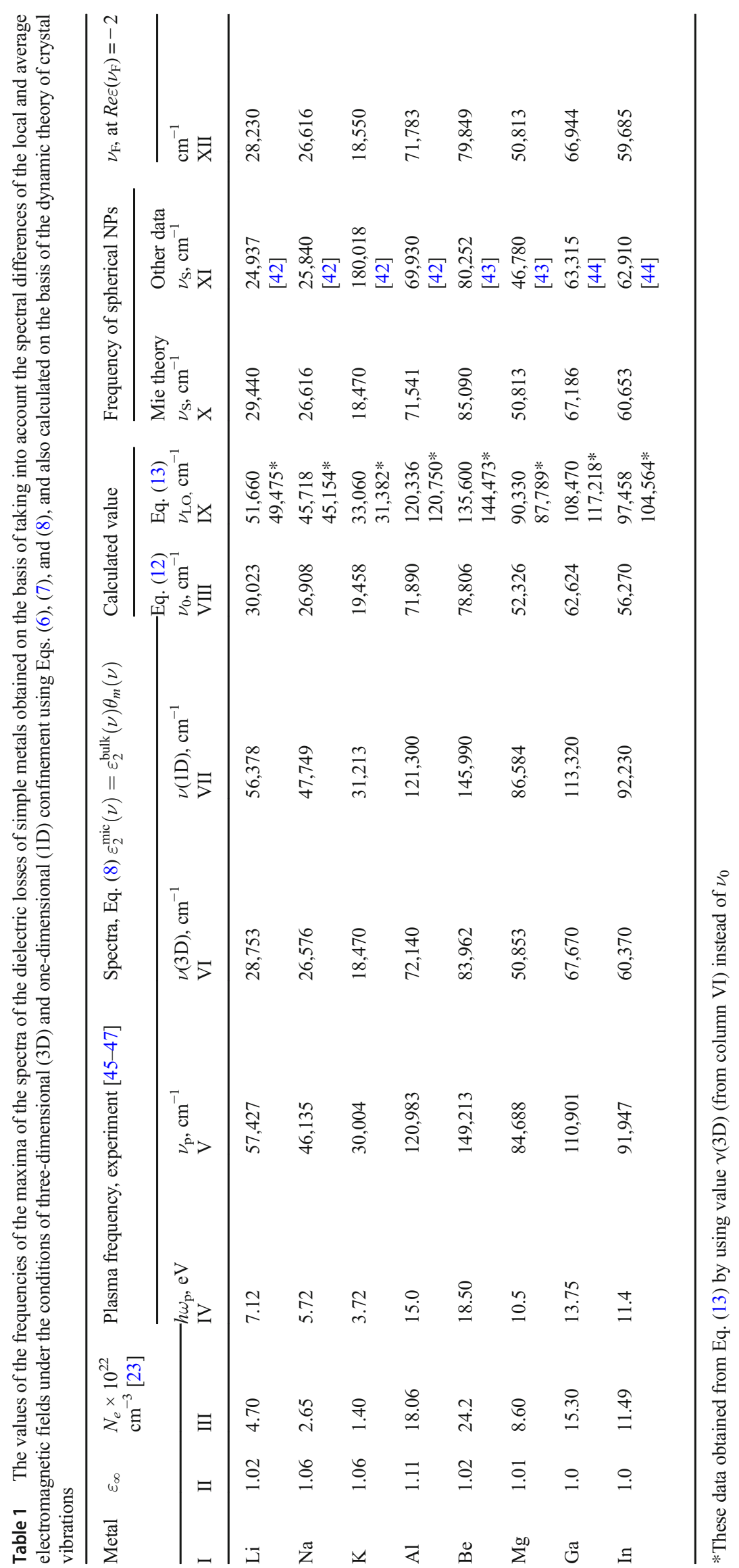


the frequencies of the maxima of spectra $\varepsilon_{2}^{\text {mic }}\left(\nu_{3 \mathrm{D}}\right)$ corresponding to three-dimensional confinement, $\nu(3 D)$, agree well with calculations of the intrinsic frequency $\nu_{0}$ from the theory of dipole-dipole interactions, using Eq. (12), and listed in column VIII.

As one would expect, the frequencies of the spectra corresponding to one-dimensional confinement $\varepsilon_{2}^{\text {mic }}\left(\nu_{1 D}\right)$ at which $\operatorname{Re} \varepsilon(\nu)=0$ are close to the plasma frequencies of the corresponding metals observed experimentally by electron energy loss spectroscopy (EELS) [45-47] and listed in column IV of Table 1. Also, the frequencies of the spectra $\varepsilon_{2}^{\text {mic }}\left(\nu_{1 D}\right)$ corresponding to the conditions required for the manifestation of the absorption bands of longitudinal oscillations of free electrons, $\nu(1 \mathrm{D})$, coincide with the frequency of the longitudinal mode $\nu_{\mathrm{LO}}$ estimated from the dynamic theory of the lattice vibrations (Eq. (13)) with an accuracy of 1-3\% (compare columns VII and IX in Table 1).

These results indicate that resonance absorption at the longitudinal vibration frequency can be observed in a thin metal layer with a thickness $h<<\lambda$, when the effect of medium polarization in the direction of oscillation is absent. This conclusion agrees with results [48] where it is shown that the appearance of absorption at the oscillation frequency LO is a consequence of the conditions existing in a thin dielectric film at an oblique incidence of the probing radiation. For metals, this corresponds to Ferrel's modes, theoretically predicted in [49] and can be observed in optical experiments using spectroscopic ellipsometry [50] as well as transmission and reflection [51-53] techniques carried out at an oblique incidence of light in a thin film. In particular, the minimum in transmission spectra obtained at the oblique incidence of p-polarized light was observed in Ref. [53] for thin films of Na and $\mathrm{K}$ at $218 \mathrm{~nm}$ $(5.7 \mathrm{eV})$ and at $326 \mathrm{~nm}(3.8 \mathrm{eV})$, respectively. In addition to that, the experimental data on optical measurements in the range of plasma frequency were also reported in early work of Ives and Briggs [54] for $\mathrm{Na}$ and $\mathrm{K}$, using a photoelectron emission from thin alkali metal films. The obtained results demonstrate a peak position at $5.9 \mathrm{eV}$ and at $3.65 \mathrm{eV}$ for $\mathrm{Na}$ and $\mathrm{K}$ respectively, which are in good agreement with experimental data on EELS, cited above transmission measurements and our calculations for $\nu$ (1D) shown in Table 1 .

In conclusion, we would like to note that a set of simple metals was selected here for investigation in order to avoid discussion of the effect of interband transitions on the peak position of the spectrum $\varepsilon_{2}^{\text {mic }}\left(\nu_{1 \mathrm{D}}\right)$ in particular. In the case of simple metals, the interband transitions are located far apart of free electron oscillation and do not affect the shape and the peak position of the spectrum of longitudinal phonons (or the bulk plasma peak $\omega_{\mathrm{p}}$ ) as can be seen from Figs. 1 and 2 and Table 1. However, the effect of closely located interband transitions on the position of plasma peak is significant in the case of the noble metals. For example, for silver and gold the $\omega_{\mathrm{p}}$ in accordance with simple Drude calculations, using the equation $\omega_{p}=\left(e^{2} N_{e} / \varepsilon_{0} m_{c}\right)^{1 / 2}$, must appear at around $9 \mathrm{eV}$. However, the LO mode was observed in Refs. [52, 55] in $p$-polarized reflection and transmission spectra, measured at oblique incidence angle from thin silver films, at $\sim 3.8 \mathrm{eV}$. Our calculation of the spectrum $\varepsilon_{2}^{\mathrm{mic}}\left(\nu_{1 \mathrm{D}}\right)$ for Ag, using data on optical constants from Ref. [56], show the peak position at $3.78 \mathrm{eV}$ in good correspondence with the experimental data of Refs. [52, 55]. In gold, $\varepsilon_{2}(\nu)$ is comparatively large in the region of $\omega_{\mathrm{p}}$ and the LO mode is strongly damped and practically could not be seen in optical experiments [50]. It is also not pronouncedly visible in our calculated spectra $\varepsilon_{2}^{\operatorname{mic}}\left(\nu_{1 \mathrm{D}}\right)$ for gold.

\section{Conclusion}

The effect of dipole-dipole interactions of free electrons was analyzed using Drude theory and the model of the Lorentz local field for a number of simple metals. The optical constant of the bulk metal was used to account for the dispersion of the local field under conditions of one-dimensional (1D) and three-dimensional (3D) confinement. The latter, expressed as a function $\varepsilon_{2}{ }^{\mathrm{mic}}\left(\nu_{3 \mathrm{D}}\right)$, corresponds to the spectra of the dielectric loss of small spherical particles with a diameter, $d$, much smaller than the wavelength of the probing radiation $(d<<\lambda)$ and demonstrates excellent agreement with the results of Mie theory and experimental data. The former, expressed as $\varepsilon_{2}{ }^{\mathrm{mic}}\left(\nu_{1 \mathrm{D}}\right)$, allows the determination of spectral microcharacteristics in the frequency region of the longitudinal collective motion of the free electrons, which corresponds to the spectra of dielectric losses of bulk plasma oscillations. To demonstrate the effect of dipole-dipole interactions for 1D confinement more clearly, we selected a number of simple metals, viz., $\mathrm{Li}, \mathrm{Na}, \mathrm{K}, \mathrm{Al}, \mathrm{Be}, \mathrm{Mg}, \mathrm{Ga}$, and $\mathrm{In}$, with interband transitions separated widely from the plasma frequency, $\omega_{\mathrm{p}}$. The peak position of the $\varepsilon_{2}{ }^{\text {mic }}\left(\nu_{1 \mathrm{D}}\right)$ spectrum for simple metals is in good agreement with experimental results from energy loss spectroscopy and data from optical and electro-optical experiments.

For silver, in which interband transitions overlap the bulk plasma oscillations, the peak position of the calculated $\varepsilon_{2}{ }^{\mathrm{mic}}\left(\nu_{1 \mathrm{D}}\right)$ spectrum is in good agreement with experimental data from transmission and reflection measurements from thin $\mathrm{Ag}$ films at an oblique incidence of light.

To summarize, optical polarization and dipole-dipole interactions create spectral differences between the local and average electromagnetic fields, both in dielectrics and metals. Our results show that taking into account the differences in the dispersion of the average macroscopic and Lorentzian local 
fields under one-dimensional confinement makes it possible to determine the dielectric loss spectra of simple metals in the frequency range of longitudinal vibrations of free electrons, the maxima of which correspond to their bulk plasma oscillations. Note that in the case under consideration, the Lorentz local field plays the role of an electrodynamic equivalent. It accounts for dipole-dipole interactions of the microscopic metal region $(d<<\lambda)$ with its polarized environment, including the influence of the entire electron cloud. It also includes the effect of interband transitions and the positively charged crystal lattice background.

\section{References}

1. Maier SA (2007) Plasmonics: fundamentals and application. Springer, New York 224p

2. Klimov VV (2014) Nanoplasmonics: fundamentals and applications. Pan Standford Publishing, Singapore

3. Cai W, Shalaev V (2010) Optical metamaterials. Fundamentals and applications. Springer, New York

4. Rivera VAG, Silva OB, Ledemi Y, Messaddeq Y, Marega E Jr (2015) Collective plasmon-modes in gain media. Quantum emitters and plasmonic nanostructures. Springer, New York

5. Kreibig U, Volmer M (1995) Optical properties of metal clusters. Springer-Verlag, New York

6. Dionne JA, Baldi A, Baum B, Ho C-S, Jankovic V, Naik GV, Narayan T, Scholl JA, Zhao Y (2015) Localized fields, global impact: industrial applications of resonant plasmonic materials. MRS Bull 40:1138-1145

7. Pissuwan D, Valenzuela SM, Cortie MB (2006) Therapeutic possibilities of plasmonically heated gold nanoparticles. Trends Biotechnol 24:62-67

8. Pelton M, Aizpurua J, Bryant G (2008) Metal-nanoparticles plasmonics. Laser Photonics Rev 2:136-159

9. Zhang H, Demir HV, Govorov AO (2014) Plasmonic metamaterials and nanocomposites with the narrow transparency window effect in broad extinction spectra. ACS Photonics 1:822-832

10. Perez-Juste J, Pastoriza-Santos I, Liz-Marzan LM, Mulvaney P (2005) Gold nanorods: synthesis, characterisation and applications. Coord Chem Rev 249:1870-1901

11. Jain PK, Huang X, El-Saed IH, El-Saed MA (2008) Noble metals on the nanoscale: optical and photothermal properties and some applications in imaging, sensing, biology and medicine. Acc Chem Res 41:1578-1586

12. Klar TA (2007) Biosensing with plasmonic nanoparticles. In: Shalaev VM, Kawata S (eds) Nanophotonics with surface plasmonics. Elsevier, Ch. 8, pp 219-264

13. Conde J, Rosa J, Lima JC, Baptista PV (2012) Nanophotonics for molecular diagnostics and therapy applications. Int J Photoenergy Article ID 619530: 1-11 https://doi.org/10.1155/2012/619530

14. Masson J-F (2017) Surface plasmon resonance clinical biosensors for medical diagnostics. ACS Sens 2:16-30

15. Zheng YB, Kiraly B, Weiss PS, Huang TJ (2012) Molecular plasmonics for biology and nanomedicine. Nanomedicine 7:751770

16. Han G, Ghosh P, Rotello VM (2007) Functionalized gold nanoparticles for drug delivery. Nanomedicine 2:113-123
17. Govorov AO, Zhang H, Gun'ko YK (2013) Theory of photoinjection of hot plasmonic carriers from metal nanostructures into semiconductors and surface molecules. J Phys Chem C 117: 16616-16631

18. Kim S, Jin J, Kim Y-J, Park I-Y, Kim Y, Kim S-W (2008) Highharmonic generation by resonant plasmon field enhancement. Nature 453:757-760

19. Maksymov IS (2016) Magneto-plasmonic nanoantenas: basics and applications. Rev Phys 1:36-51. https://doi.org/10.1016/j.revip. 2016.03.002

20. Ordal MA, Bell RJ, Alexander RW Jr, Long LL, Querry MR (1985) Optical properties of fourteen metals in the infrared and far infrared: $\mathrm{Al}, \mathrm{Co}, \mathrm{Cu}, \mathrm{Au}, \mathrm{Fe}, \mathrm{Pb}, \mathrm{Mo}, \mathrm{Ni}, \mathrm{Pd}, \mathrm{Pt}, \mathrm{Ag}, \mathrm{Ti}, \mathrm{V}$, and W. Appl Opt 24(24):4493-4499

21. Adachi S (2012) The handbook on optical constants of metals: in tables and figures. World Scientific, Singapore

22. Rakic AD, Dijrisik AB, Elazar JM, Majewski ML (1998) Optical properties of metal films for vertical-cavity optoelectronic devices. Appl Opt 37(N28):5271-5273

23. Kittel C (1968) Introduction to solid state physics. Wiley, New York

24. Wooten F (1972) Absorption and dispersion. Optical properties of solids. Academic, New York

25. Shaganov II, Perova TS, Berwick K (2017) The effect of the local field and dipole-dipole interactions on the absorption spectra of noble metals and the plasmon resonance of their nanoparticles. Photonics Nanostruct Fundam Appl 27:24-31

26. Bakhshiev NG, Girin OP, Libov VS (1963) Relation between the observed and true absorption spectra of molecules in a solid medium. II. Methods for determining the correction of a universal effect of the effective (internal) field. Opt Spektrosk 14:745 [Opt. Spectrosc. (USSR) 14: 634]

27. Bakhshiev NG (2005) Photophysics of dipole-dipole interactions. Izd. SPbGU, St. Petersburg

28. Perova TS, Shaganov II, Vij JK, Nielsen OF, Perov PA (2000) Internal field correction of the far-infrared spectra of acetonitrile at different temperatures. Asian Chem Lett 4:191-199

29. Shaganov II, Perova TS, Moore RA (2005) Berwick effect of the internal field on the IR absorption spectra of small particles in the case of 3D, 2D and 1D size confinement. Phys Chem B 109:98859891

30. Shaganov II, Perova TS, Melnikov VA, Dyakov S, Berwick K (2010) Size effect on the infrared spectra of condensed media under condition of 1D, 2D and 3D dielectric confinement. J Phys Chem C 114:16071-16081

31. Perova TS, Shaganov II, Unnikrishnan S, Moore RA (2005) Spectroscopic characteristics of nano-composite structures in 3D, 2D and 1D size confinement. Proc SPIE 5826:387-396

32. Fröhlich H (1958) Theory of dielectrics. Oxford University Press, London

33. Shaganov II, Perova TS, Mukhina MV, Martynenko IV, Baranov AV, Fedorov AV, Gerard V, Gun'ko YK (2014) Influence of intermolecular interactions on spectroscopic characteristics of metal nanoparticles and their composites. Phys Chem Chem Phys 16: 24536-24548

34. Born M, Wolf E (1999) Principles of optics, 7th edn. Elsevier, Cambridge

35. Born M, Huang K (1954) Dynamical theory of crystal lattice. London, Oxford

36. Bonnevile R (1980) Local-field factors in cubic crystals. Phys Rev B 21(N2):368-376

37. Wang Y, Plummer EW, Kempa K (2011) Foundations of Plasmonics. Adv Phys 60(N5):799-898

38. Palik ED (ed) (1998) Handbook of optical constants of solids. Academic Press, San Diego

39. Palik ED (ed) (1991) Handbook of optical constants of solids II. Academic Press, San Diego 
40. Hagemann H-J, Gudat W, Kunz C (1975) Optical constants from the far infrared to the $\mathrm{x}$-ray region: $\mathrm{Mg}, \mathrm{Al}, \mathrm{Cu}, \mathrm{Ag}, \mathrm{Au}, \mathrm{Bi}, \mathrm{C}$, and $\mathrm{Al}_{2} \mathrm{O}_{3}$. J Opt Soc Am 65:742-744

41. Jezequel G, Lemonnier JC, Thomas J (1977) Optical properties of gallium films between 2 and $15 \mathrm{eV}$. J Phys F Met Phys 7:16131622

42. Blaber MG, Arnold MD, Harris N, Ford MJ, Cortie MB (2007) Plasmon absorption in nanospheres: a comparison of sodium, potassium, aluminium, silver and gold. Physica B 394:184-187

43. Sanz JM, Ortiz D, de la Osa RA, Saiz JM, Gonzalez F, Brown AS, Losurdo M, Everitt HO, Moreno F (2013) UV plasmonic behaviour of various metal nanoparticles in the near- and far-field regimes: geometry and substrate effects. J Phys Chem C 117:19606-19615

44. McMahon JM, Schatz GC, Gray SK (2013) Plasmonics in the ultraviolet with the poor metals. PCCP 15:5415-5423

45. Powell CJ (1960) The origin of the characteristic electron energy losses in ten elements. Proc Phys Soc 76:593-610

46. Raether H (1980) Excitation of plasmons and interband transitions by electrons. In: Höhler G (ed) Springer tracts in modern physics, vol 88. Springer-Verlag, Berlin-Heidelberg $196 \mathrm{p}$

47. Strawbridge B, Singh RK, Beach C, Mahajan S, Newman N (2006) Effect of surface topography on reflection electron energy loss plasmon spectra of group III metals. J Vac Sci Technol A 24:1776-1781

48. Lehmann A (1988) Theory of infrared transmission spectra of thin insulating films. Phys Status Solidi A 148:401-405
49. Ferrel RA (1958) Predicted radiation of plasma oscillations in metal films. Phys Rev 111:1214-1222

50. Oates TWH, Wormeester H, Arwin H (2011) Characterisation of plasmonic effects in thin films and metamaterials using spectroscopic ellipsometry. Prog Surf Sci 86:328-376

51. Berreman DW (1963) Infrared absorption at longitudinal optic frequency in cubic crystal films. Phys Rev 130:2193-2198

52. McAlister AJ, Stern EA (1963) Plasma resonance absorption in thin metal films. Phys Rev 132:1599-1602

53. Melnyk AR, Harrison MJ (1970) Theory of optical excitations of plasmon in metals. Phys Rev B 2:835-850

54. Ives HE, Briggs HB (1938) Correlation of optical properties and photoelectric emission in thin films of alkali metals. J Opt Soc Am 28:330-338

55. Bichri A, Lafait J, Welsch H (1993) Visible and infrared optical properties of $\mathrm{Ag} / \mathrm{SiO}_{2}$ multilayers: radiative virtual modes and coupling effects. J Phys Condens Matter 5:7361-7374

56. Stahrenberg K, Herrmann T, Wilmers K, Esser N, Richter W (2001) Optical properties of copper and silver in the energy range 2.5-9.0 eV. Phys Rev B 64:115111/1-115111/9

Publisher's Note Springer Nature remains neutral with regard to jurisdictional claims in published maps and institutional affiliations. 